\title{
analyse de la stabilité des ouvrages en sols renforcés par une méthode d'homogénéisation
}

\author{
stability analysis of reinforced soil structures \\ through a homogenization method
}

\author{
Patrick de BUHAN et Jean SALENCCON \\ Laboratoire de Mécanique des Solides* \\ (Centre commun E.P., E.N.S.M. Paris, \\ E.N.P.C., associé au C.N.R.S.)
}

\section{Résumé}

L'emploi de plus en plus fréquent des techniques de renforcement des sols dans la construction géotechnique, nécessite l'élaboration de méthodes de dimensionnement à la rupture des ouvrages ainsi renforcés, qui soient à la fois fiables et simples à utiliser. La méthode ici proposée part de l'idée intuitive selon laquelle le sol renforcé peut à l'échelle de l'ouvrage, être considéré comme un matériau homogène, mais anisotrope en raison de l'orientation privilégiée des inclusions de renforcement.

Le critère de résistance d'un tel matériau homogène équivalent est déterminé par voie théorique à partir de ceux des constituants du sol renforcé. Un tel critère, que l'on peut explicitement construire dans le cas d'une modélisation multicouche du sol renforcé, est alors appliqué à l'analyse de stabilité de queiques ouvrages, en particulier dans le cas de la terre armée où les évaluations théoriques obtenues se révèlent en bon accord avec les résultats expérimentaux. En dépit de certaines limitations qui sont évoquées dans l'article, il apparaît que l'approche par homogénéisation en calcul à la rupture ainsi proposée est susceptible de constituer une méthode de dimensionnement efficace pour les ouvrages en sols renforcés.

\section{Abstract}

The increasing use of soil reinforcement techniques in the field of geotechnical engineering, requires the elaboration of reliable as well as practical yield design procedures for reinforced soil structures. The method presented hereafter, originates from the intuitive idea that from a macroscopic point of view, reinforced soils can be regarded as homogeneous but anisotropic materials, on account of the existence of privileged orientations due to the reinforcing inclusions.

The strength criterion of such an equivalent homogeneous material can be theoretically determined starting from the strength characteristics of the reinforced soil components. Application of such a criterion, which can be explicitely formulated within the framework of a multilayered modelization for the reinforced soil, is then performed on the case of the stability analysis of some typical structures. Special concern has been given to reinforced earth structures, and it turns out that the theoretical estimations so obtained are in good agreement with experimental data. Despite some limitations which are outlined in the paper, the yield design homogenization procedure thus proposed is likely to become an appropriate design method for reinforced soil structures. 


\section{INTRODUCTION}

Depuis l'invention de la terre armée par H. VIDAL il y a maintenant plus de vingt ans, les procédés de renforcement des sols se sont considérablement développés à travers le monde dans le domaine de la construction géotechnique. Ils se sont progressivement imposés comme des méthodes à la fois simples de mise en cuvre et économiquement avantageuses par rapport à d'autres solutions plus traditionnelles qui permettent d'améliorer les propriétés mécaniques des sols. Pardelà l'extrême diversité des techniques utilisées (terre armée, clouage, traitement par colonnes, géotextiles...), qui sont fonctions tant du type d'ouvrage que l'on cherche à réaliser, que de la nature des terrains rencontrés, ces procédés se caractérisent tous par l'introduction dans le sol naturel d'éléments de structure appelés inclusions, destinés à accroître les capacités de résistance de l'ouvrage, et donc à en assurer la tenue sous l'action des charges qu'il est amené à supporter.

Il en résulte l'apparition d'un "matériau composite " de conception originale, formé par l'association du sol et des inclusions de renforcement. Or si l'élaboration et la mise en ceuvre d'un tel matériau dans la construction des ouvrages, sont à l'heure actuelle assez bien maîtrisées d'un point de vue technologique, il n'en est pas encore de même pour ce qui est des méthodes de calcul et de dimensionnement correspondantes.

Bien entendu, de nombreuses méthodes existent d'ores et déjà qui permettent d'analyser la stabilité des ouvrages en sols renforcés. On en trouvera une description détaillée dans les comptes rendus des deux colloques internationaux sur les techniques de renforcement des sols, qui se sont déroulés à Paris en 1979 et 1984, ainsi qu'à travers les nombreuses communications consacrées à cette question dans le cadre du $\mathrm{Xl}^{\text {e }}$ congrès international de Mécanique des Sols. Adaptées pour la plupart d'entre elles de l'approche désormais classique pour les ouvrages en sols homogènes, qui consiste à vérifier leur stabilité vis-à-vis de surfaces de rupture potentielles, elles s'efforcent de prendre en compte de diverses manières, dans le bilan des efforts résistants, le caractère composite du sol renforcé (voir par exemple [JURAN et SCHLOSSER - 1979]. [BLONDEAU et al. - 1984], [DELMAS et al. - 1986], [RENOUF et al. - 1982], [LESHCHINSKY et REINSCHMIDT - 1985]). Mais la mise en ceuvre de ces méthodes repose le plus souvent sur des hypothèses simplificatrices concernant la façon dont les inclusions de renforcement interagissent avec le sol environnant. L'analyse de certaines d'entre elles du point de vue mécanique, ainsi que des hypothèses qui les soustendent [de BUHAN - 1986], montre qu'elles peuvent conduire soit à surestimer, parfois de façon importante, les capacités de résistance du sol renforcé et donc la stabilité des ouvrages concernés, soit au contraire à surdimensionner ces derniers.

Une autre voie d'approche consiste à procéder à une analyse complète du problème par l'emploi de méthodes numériques de type "éléments finis». C'est le sens des travaux de [CORTE - 1977], [BROWN et POULOS - 1979], ou [BASTICK - 1987] sur la terre armée, ainsi que de ceux de [JURAN et al, - 1985] sur les sols renforcés par clouage. Il convient néanmoins de remarquer que l'objectif de ces travaux n'est pas tant d'étudier la stabilité de ces ouvrages, que de mieux mettre en lumière leur fonctionnement dans une phase préalable à la rupture, et en particulier la façon dont les efforts internes se redistribuent dans le massif entre le sol et les armatures. Ce n'est que très récemment qu'a été abordé de manière explicite le problème de la stabilité des ouvrages en sols renforcés par une méthode des éléments finis formulée dans le cadre de l'analyse limite [CISS -1985], [PASTOR et al.- 1986]. L'une des principales conclusions que lon peut tirer de ces derniers travaux, actuellement en cours de développement, est, qu'en raison du caractère fortement hétérogène du matériau constitutif, il se révèle beaucoup plus difficile de calculer les chargements limites dans le cas d'ouvrages en sols renforcés que dans le cas d'ouvrages homogènes.

L'approche présentée dans cet article vise à proposer une nouvelle méthode de dimensionnement pour les ouvrages en sols renforcés, fondée d'une part sur la théorie du calcul à la rupture, conçue comme une généralisation de l'analyse limite [SALENÇON -1983], d'autre part sur les théories relatives à l'homogénéisation des milieux hétérogènes périodiques et plus particulièrement l'application qui en a été faite aux sols renforcés [de BUHAN - 1986]. Dans son principe cette méthode repose tout simplement sur l'intuition selon laquelle, à l'échelle des dimensions des ouvrages, les sols renforcés peuvent être perçus comme des milieux homogènes anisotropes, dont les caractéristiques de résistance mécanique peuvent être déterminées à partir de celles des constituants (sol et renforcements). La méthode d'homogénéisation en calcul à la rupture développée à partir de cette intuition, est alors appliquée à l'analyse de stabilité de quelques ouvrages types dans lesquels le sol renforcé est modélisé comme un matériau multicouche.

\section{FONDEMENTS GÉNÉRAUX D'UNE MÉTHODE D'HOMOGÉNÉISATION APPLIQUÉE AUX SOLS RENFORCÉS}

\subsection{Les sols renforcés vus sous l'angle de milieux homogènes anisotropes}

L'idée d'employer une méthode d'homogénéisation pour l'étude des sols renforcés n'est à vrai dire pas tout à fait nouvelle. On la trouve présente par exemple chez [GERRARD - 1982], [GERRARD et al. - 1984], et elle constitue même le fil directeur des travaux de SAWICKI sur la terre armée depuis plusieurs années [SAWICKI - 1979, 1983].

La possibilité d'homogénéiser un sol renforcé, c'est-àdire de l'assimiler d'une certaine façon à un matériau homogène « équivalent », suppose en fait remplies trois conditions.

a) Il convient de souligner en tout premier lieu, que le concept d'homogénéisation d'un sol renforcé ne revêt de sens que dans la mesure où l'on s'intéresse non pas 
à ce qui peut se passer localement en tel ou tel point de l'ouvrage, mais à l'évaluation de grandeurs globales qui se rattachent à celui-ci. Tel est précisément le cas des analyses de stabilité qui visent à estimer par le calcul les chargements pour lesquels la ruine d'un ouvrage dans son ensemble risque de se produire.

b) Il importe ensuite que les inclusions de renforcement soient réparties dans le sol de façon à peu près régulière, de sorte que l'on puisse alors modéliser le sol renforcé comme un matériau composite à structure périodique. Cette condition de périodicité apparait le plus souvent vérifiée, exception faite par exemple pour des sols renforcés de manière aléatoire ("Texsol» [LEFLAIVE - 1985]) qui sortent du cadre de notre étude.

c) Il est essentiel enfin que la période caractéristique du renforcement (l'espacement vertical entre deux nappes horizontales successives d'armatures par exemple dans un ouvrage en terre armée) puisse être considérée comme petite vis-à-vis des dimensions d'ensemble de l'ouvrage. Cette dernière condition est en pratique plus difficilement satisfaite, en raison du nombre parfois assez peu significatif des inclusions incorporées dans le sol.

Dans ces conditions, il apparaît clairement que la structure géométrique particulière du sol renforcé et notamment l'existence d'orientations privilégiées liée aux inclusions de renforcement, conférera au matériau * sol renforcé » un caractère anisotrope.

La méthode d'homogénéisation proposée dans cet article découle de l'idée intuitive que, sous réserve que les conditions énoncées précédemment soient satisfaites, il est possible de procéder à l'analyse de stabilité d'un ouvrage en sol renforcé en considérant un "ouvrage homogène associé» (figure 1), de même géométrie que l'ouvrage réel initial, soumis aux mêmes conditions de chargement, mais dans lequel le sol renforcé, de nature composite, est remplacé par un matériau homogène anisotrope. L'analyse de stabilité étant menée dans l'esprit du calcul à la rupture, c'est-à-dire à partir de la seule donnée du critère de résistance du matériau constitutif de l'ouvrage examiné, on voit que la méthode d'homogénéisation dépend fondamentalement de la possibilité de définir un critère de résistance macroscopique qui permette de caractériser les capacités de résistance du sol renforcé considéré en tant que matériau homogène.

OUVRAGE INITIAL

EN SOL RENFORCÉ

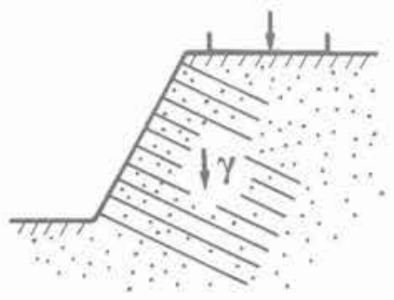

OUVRAGE HOMOGENE ASSOCIÉ

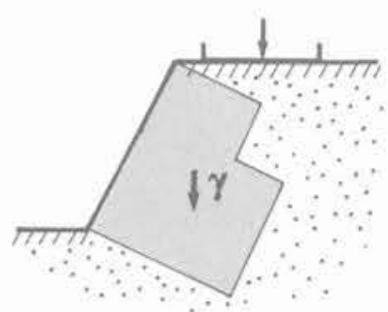

Fig. 1. - Principe de la méthode d'homogénéisation.

Fig. 1. - A sketch of the homogenization method.
Il convient de remarquer, comme cela est suggéré sur la figure 1, qu'une telle procédure d'homogénéisation ne porte que sur les zones de l'ouvrage effectivement renforcées, les autres parties demeurant inchangées dans la définition du problème homogène associé.

\subsection{Méthode d'homogénéisation en calcul à la rupture}

Ayant ainsi esquissé dans ses grandes lignes le principe d'une méthode d'homogénéisation qui s'applique à l'étude de stabilité des ouvrages en sols renforcés, il convient à présent d'en donner une description plus précise fondée sur une analyse mécanique rigoureuse du problème. Celle-ci nous est fournie par la théorie du calcul à la rupture [SALENÇON - 1983] à laquelle nous nous référerons constamment par la suite. Laissant au lecteur soucieux de plus de précision le soin de se reporter à [de BUHAN - 1986] pour les fondements théoriques de la méthode, nous nous bornerons ici à en exposer les principaux résultats.

La mise en œuvre de la méthode d'homogénéisation comporte deux étapes distinctes :

- la détermination du critère de résistance macroscopique du sol renforcé à partir de la connaissance des capacités de résistance de ses constituants;

- la résolution du problème homogène associé fondée sur l'utilisation de ce critère.

\subsubsection{Détermination du critère de résistance macroscopique}

Il ne saurait évidemment être question d'obtenir un tel critère par voie expérimentale ${ }^{*}$, sauf à imaginer que l'on puisse effectuer des essais sur des volumes de sol renforcé qui, pour être représentatifs, devraient être de l'ordre de plusieurs mètres cubes! Une approche théorique se révèle donc nécessaire.

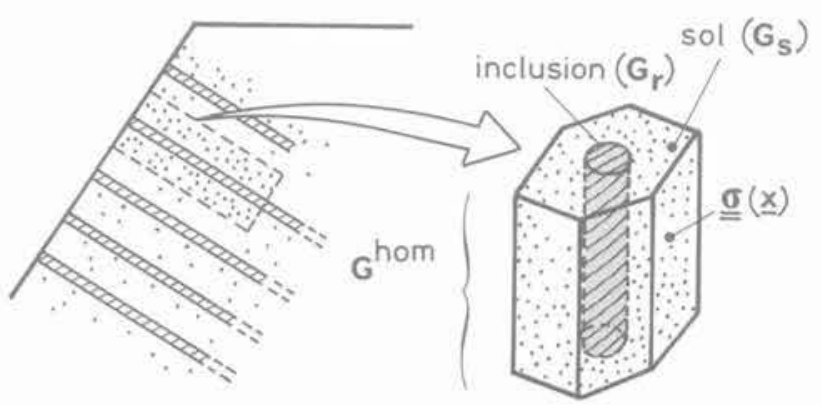

Fig. 2. - Cellule de base représentative d'un sol
renforcé de manière périodique.

Fig. 2. - Representative cell for a periodically reinforced soil.

\footnotetext{
- A l'exception notable de la terre armée, où des essais au " triaxial » sur des éprouvettes de sable armées par des disques métalliques permettent d'évaluer, au moins pour des états de contrainte particuliers, les capacités de résistance de ce matériau (voir [LONG et al. - 1972], et paragraphe 4.2.).
} 
La propriété de périodicité du renforcement joue de ce point de vue un rôle essentiel. Elle permet en effet de mettre en évidence une cellule de base représentative du sol renforcé qui comporte généralement une seule inclusion (figure 2). La construction du critère découle alors de la résolution d'un problème de calcul à la rupture défini sur cette cellule de base de la manière suivante.

Désignant par $G_{s}$ (respectivement $G_{s}$ ) le convexe de résistance du sol (respectivement du matériau qui constitue les inclusions de renforcement), c'est-à-dire le domaine des contraintes $\cong$ admissibles, et supposant que le contact entre le sol et l'inclusion est à adhérence totale, on montre que le domaine, noté Ghom, qui caractérise le critère de résistance macroscopique du sol renforcé est formé par l'ensemble des états de

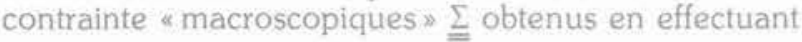
la moyenne volumique sur la cellule de base de tous les champs de contrainte ₹ périodiques, en équilibre, et respectant le critère de résistance en tout point. Soit :

$$
\sum_{\underline{i}} \in G^{\text {hom }}
$$

Il existe $\cong$ périodique tel que :

$\underline{\underline{\Sigma}}=\langle\underline{\underline{\sigma}}\rangle\rangle^{*}, \operatorname{div} \underline{\underline{\sigma}}=0$, et en tout point $\underline{\underline{x}}, \underline{\underline{g}}(\underline{\mathrm{x}}) \in \mathrm{G}$, ou $G$, selon que le point $x$ appartient au sol ou à l'inclusion de renforcement.

On voit immédiatement que, du fait de la convexité des domaines $G_{s}$ et $G_{T}$, le domaine $G^{\text {hom }}$ ainsi défini est lui-même convexe.

- \langle\rangle désigne l'opération de moyenne volumique sur la cellule de base représentative.

\subsubsection{Résolution du problème homogène associé}

Elle consiste à mettre en cuvre, sur l'ouvrage homogène associé, les approches classiques du calcul à la rupture dont nous rappelons ci-dessous brièvement le principe. Nous nous restreindrons ici, pour simplifier. au cas où l'ouvrage est soumis à un chargement dépendant d'un seul paramètre Q

- Approche statique « par l'intérieur».

L'ouvrage sera dit potentiellement stable sous le chargement $Q$ s'il est possible d'équilibrer $Q$ par un champ de contrainte $\underline{\sigma}$ tel que :

$$
\underline{\underline{g}}(\underline{\mathrm{x}}) \in \mathrm{G}^{\text {hom }} \text { en tout point } \underline{\mathrm{x}} \text {. }
$$

et on désignera alors par $\mathrm{Q}^{\text {hom }}$ la valeur extrême du chargement définie par :

$\mathrm{Q} \leqslant \mathrm{Q}^{\text {hom }} \Leftrightarrow$ Ouvrage potentiellement stable sous $\mathrm{Q}$.

L'utilisation de l'approche statique conduit à l'obtention d'un minorant de $\mathrm{Q}^{\text {hom }}$.

\section{- Approche cinématique «par l'extérieur».}

Considérant un champ de vitesse $\underline{v}$ cinématiquement admissible quelconque défini en tout point de l'ouvrage, on peut écrire l'inégalité suivante relative au chargement extrême $\mathrm{Q}^{\text {hom : }}$

$$
P\left(Q^{\text {hom }} \cdot \underline{v}\right) \leqslant P^{\text {hom }}(\underline{v})
$$

où $P\left(Q^{\text {hiom, }}, \underline{v}\right)$ représente la puissance des efforts extérieurs à l'ouvrage calculée dans le champ v, et $\mathrm{P}$ hom (v) est une fonctionnelle à valeurs positives définie par :

$\mathrm{P}^{\operatorname{hom}}(\underline{\mathrm{v}})=\int_{V} \pi^{\text {hom }}(\underline{\mathrm{d}}(\underline{\mathrm{x}})) \mathrm{dV}+\int_{\Sigma} \pi^{\text {hom }}(\underline{\mathrm{n}}(\underline{\mathrm{x}}) ;[\underline{\mathrm{v}}(\underline{\mathrm{x}})]) \mathrm{d} \Sigma$ avec :

- $\underline{\underline{d}}$ : le champ de vitesse de déformation associé à $\underline{v}$ :

$$
\mathrm{d}_{i j}=1 / 2\left(\partial \mathrm{v}_{i} / \partial \mathrm{x}_{1}+\partial \mathrm{v}_{j} / \partial \mathrm{x}_{i}\right) ;
$$

- $[\underline{v}(\underline{x}) \rrbracket$ : la valeur de la discontinuité du champ $\underline{v}$ au franchissement d'une éventuelle surface de discontinuité $\Sigma$ dans le sens de la normale $\underline{n}$ au point $\underline{x}$;

- et par définition :

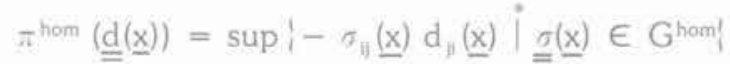

et

$$
\begin{aligned}
& \pi^{\text {hom }}(\underline{\mathrm{n}}(\underline{\mathrm{x}}) ; \llbracket \underline{\mathrm{v}}(\underline{\mathrm{x}}) \rrbracket)=\sup ;-\sigma_{\mathrm{ij}}(\underline{\mathrm{x}}) \mathrm{n}_{1}(\underline{\mathrm{x}}) \llbracket \mathrm{v}_{1}(\underline{\mathrm{x}}) \rrbracket \\
& \underline{\underline{g}(\mathrm{x})} \in \mathrm{G}^{\text {hom }} \text {. }
\end{aligned}
$$

L'utilisation de lapproche cinématique conduit à l'obtention d'un majorant de Q ${ }^{\text {hom }}$.

- Sommation sur les indices répétés; les contraintes de compression sont comptées positivement.

\subsection{Domaine de validité de la méthode}

Nous avons déjà fait observer que l'applicabilité de la méthode d'homogénéisation ainsi proposée au calcul de stabilité des ouvrages en sols renforcés reposait sur un certain nombre de conditions : caractère périodique du renforcement (condition essentielle qui permet d'obtenir une formulation théorique du critère de résistance macroscopique), nombre suffisant d'inclusions de renforcement permettant de conférer au matériau composite ainsi obtenu une certaine «homogénéité macroscopique». Reste alors à savoir, en admettant que de telles conditions soient satisfaites, quel est le lien entre la solution du problème de calcul à la rupture sur l'ouvrage homogène associé et celle du problème initial. Un résultat d'homogénéisation de portée très générale établi par SUQUET [1983] puis de BUHAN [1986] permet de répondre partiellement à cette question. Il peut s'énoncer ainsi :

"L'ouvrage homogène (ainsi) associé est plus stable que l'ouvrage réel initial, „

Ce que l'on peut traduire par l'inégalitê

$$
Q^{\text {hom }} \geqslant Q^{*}
$$


dans laquelle $Q^{*}$ désigne le chargement extrême au-delà duquel l'instabilité de l'ouvrage initial est certaine.

L'inégalité inverse (qui impliquerait alors l'équivalence complète entre le problème homogène associé et le problème initial) ne peut être prouvée. Il est même possible de mettre en évidence des exemples pour lesquels les valeurs de $Q^{*}$ et $Q^{\text {hom }}$ se révèlent être significativement différentes [de BUHAN - 1986]. Cette différence peut notamment être attribuée, semble-t-il. à ce qu'il est convenu d'appeler les "effets de bord ». Ce sont bien d'une certaine façon ces effets qui sont explicitement pris en compte dans la pratique lors de la construction des ouvrages de soutènement en terre armée, par la mise en place d'une "peau " dont la fonction est précisément d'éviter l'apparition de désordres qui résulteraient de l'écoulement du matériau de remblai (sable sec sans cohésion : voir quatrième partie) entre les lits d'armatures à proximité du parement extérieur.

On peut donc dire qu' " aux effets de bord près *, c'està-dire à la condition de négliger ceux-ci ou plus exactement de les maitriser par des dispositions constructives adéquates, l'étude de stabilité d'un ouvrage en sol renforcé se ramène bien à celle de l'ouvrage homogène associé.

\section{UN PREMIER MODÈLE DE SOL RENFORCÉ : LE MATÉRIAU MULTICOUCHE PUREMENT COHÉRENT}

Peu représentatif des modes de renforcement des sols par clouage ou par l'introduction de pieux ou de colonnes, le modèle de matériau multicouche constitue en revanche une schématisation tout à fait convenable du renforcement des sols par inclusions disposées en nappes. Ayant en vue l'application à des problèmes de calcul à la rupture "en déformation plane ", nous étudierons ce modèle dans le formalisme du milieu continu bidimensionnel.

\subsection{Un critère macroscopique de type cohérent anisotrope}

Le sol renforcé, modélisé comme un matériau multicouche bidimensionnel, est repéré dans les axes Oxy. l'axe Ox étant pris parallèle à l'orientation des couches (figure 3). On suppose que le sol, ainsi que le matériau

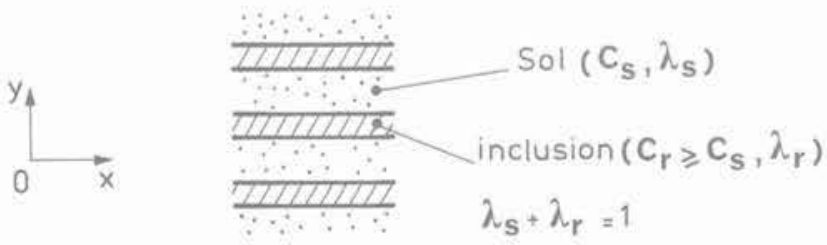

Fig. 3. - Modèle de matériau multicouche à constituants purement cohérents.

Fig. 3. - A multilayered material with purely cohesive constituents. de renforcement sont des milieux homogènes isotropes purement cohérents, de cohésions respectives $C_{\text {s }}$ et $C$,

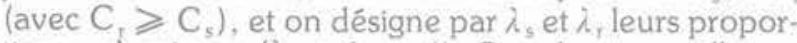
tions volumiques $\left(\lambda_{1}+\lambda_{2}=1\right)$. On admet par ailleurs, au moins dans un premier temps, que le contact entre les deux constituants est à adhérence totale sans décollement possible.

Partant de la définition générale (1), on peut alors montrer [de BUHAN - 1986] que le convexe $G^{\text {hom }}$ (domaine dans l'espace des contraintes bidimensionnelles) qui caractérise les capacités de résistance du matériau multicouche à l'échelle macroscopique est défini par :

$$
\underline{\underline{\underline{\Sigma}}} \in \mathrm{G}^{\mathrm{hom}} \Leftrightarrow \underline{\underline{\Sigma}}=\lambda_{\mathrm{s}} \underline{\underline{\underline{\sigma^{s}}}}+\lambda_{\mathrm{r}} \underline{\underline{\underline{\sigma^{t}}}}
$$

où les contraintes $\underline{\underline{\sigma^{5}}}$ et $\underline{\underline{\sigma^{r}}}$ vérifient les conditions de résistance respectives du sol et du matériau de renforcement, qui s'expriment comme suit en fonction des contraintes principales dans le plan Oxy :

$$
\begin{aligned}
& \sigma_{1}^{s}-\sigma_{2}^{s} \leqslant 2 C_{s} \\
& \sigma_{1}^{t}-\sigma_{2}^{t} \leqslant 2 C_{t}
\end{aligned} \quad\left(\sigma_{1} \geqslant \sigma_{2}\right)
$$

ainsi que les relations :

$$
\begin{aligned}
& \sigma_{x y}^{s}=\sigma_{x y}^{y}=\Sigma_{x y} \\
& \sigma_{y y}^{s}=\sigma_{y y}^{y}=\Sigma_{y y}
\end{aligned}
$$

qui traduisent la continuité du vecteur-contrainte à l'interface entre les couches.

La combinaison des relations (5), (6) et (7) permet d'écrire le critère de résistance macroscopique sous la forme plus condensée

$$
\Sigma_{1}-\Sigma_{2} \leqslant 2 C(\alpha)
$$

où $\Sigma_{1} \geqslant \Sigma_{2}$ sont les composantes principales de la contrainte macroscopique $\Sigma$, et $\alpha$ l'angle que fait la contrainte principale majeure $\Sigma_{1}$ avec la direction $\mathrm{Oy}$.

$\mathrm{C}(\alpha)$ a la dimension d'une cohésion : son diagramme polaire est représenté sur la figure 4. Elle peut s'écrire sous la forme:

$$
C(\alpha)=C_{s} \cdot \rho\left(\alpha: \lambda_{T}, C_{r} / C_{s}\right)
$$

où $\rho$ est un facteur sans dimension dont on peut expliciter l'expression en fonction des arguments ci-dessus.

Le critère ainsi obtenu est du type "cohérent aniso. trope ». La cohésion anisotrope $\mathrm{C}(\alpha)$ passe par un minimum égal à $\mathrm{C}_{\mathrm{s}}$ (cohésion du sol non renforcé) pour $\alpha=\pi / 4$ et devient maximale lorsque les directions principales de la contrainte $\Sigma$ coïncident avec les axes Ox et $\mathrm{Oy}$ :

$$
\mathrm{C}(\alpha=0)=\mathrm{C}(\alpha=\pi / 2)=\lambda_{s} \mathrm{C}_{s}+\lambda_{r} \mathrm{C}_{r} .
$$

Ce maximum n'est autre que la moyenne des cohésions des constituants du sol renforcé pondérée par leurs proportions volumiques. 


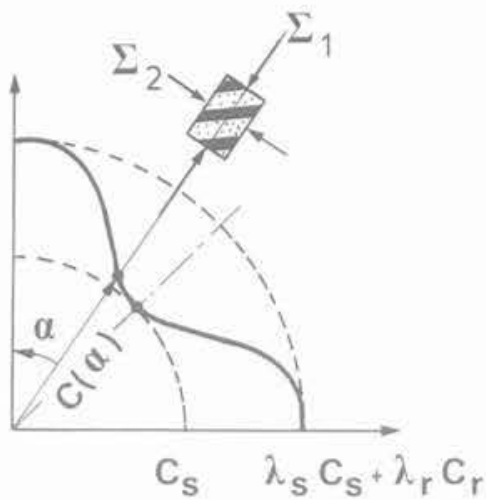

Fig. 4. - Diagramme polaire de cohésion du sol renforcé multicouche.

Fig. 4. - Polar diagram for the cohesion of a multilayer reinforced soil.

Nous nous proposons maintenant d'appliquer le critère ainsi déterminé à l'analyse de stabilité d'une pente en sol renforcé d'une part, et au calcul de la capacité portante d'une fondation sur sol renforcé d'autre part.

\subsection{Analyse de stabilité d'une pente en sol renforcé [de BUHAN et SALENÇON - 1985]}

On s'intéresse au problème de la stabilité sous poids propre d'un talus de pente $\beta$ et de hauteur h, reposant sur un substratum supposé infiniment rigide, et constitué du sol renforcé multicouche décrit précédemment (figure 5).

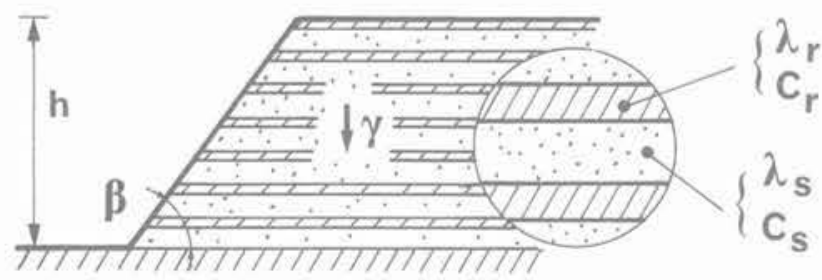

Fig. 5. - Stabilité sous poids propre d'un talus en sol renforcé multicouche.

Fig. 5. - Slope stability analysis for a reinforced soil embankment.

Désignant par $\gamma$ le poids volumique du sol, que l'on supposera pour simplifier identique à celui du matériau de renforcement, on sait que la stabilité d'un tel ouvrage est gouvernée par le facteur sans dimension :

$$
\mathrm{K}=\gamma \mathrm{h} / \mathrm{C}_{\mathrm{s}}
$$

qui doit demeurer inférieur à une valeur critique, notée $\mathrm{K}^{*}$, fonction uniquement de l'angle $\beta$ ainsi que des arguments sans dimension $C_{r} / C_{s}$ et $\lambda_{r}\left(\right.$ ou $\left.\lambda_{s}\right)$ :

$\gamma \mathrm{h} / \mathrm{C}_{\mathrm{s}} \leqslant \mathrm{K}^{*} \Leftrightarrow$ Talus potentiellement stable

$$
\mathrm{K}^{*}=\mathrm{K}^{*}\left(\beta, \mathrm{C}_{\mathrm{t}} / \mathrm{C}_{\mathrm{s}}, \lambda_{\mathrm{t}}\right) \text {. }
$$

\subsubsection{Calcul par la méthode d'homogénéisation}

L'ouvrage homogène associé au talus initial en sol ren- forcé est un talus de même géométrie (h, $\beta$ ), constitué d'un matériau homogène de poids volumique $\gamma$ obéis sant au critère de résistance anisotrope (8) (figure 6)

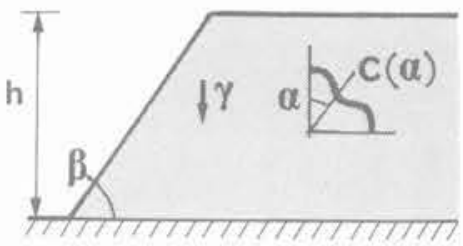

Fig. 6. - Talus homogène anisotrope associé. Fig. 6. - The anisotropic homogeneous embankment associated to fig. 5.

Compte tenu de l'expression (9) de la cohésion anisotrope $C(\alpha)$, il apparaît que la stabilité de cet ouvrage est également conditionnée par le facteur sans dimension $\gamma \mathrm{h} / \mathrm{C}_{\mathrm{s}}$. Désignant par $\mathrm{K}^{\text {hom }}$ la valeur extrême de ce facteur, on peut écrire :

$$
\gamma \mathrm{h} / \mathrm{C}_{\mathrm{s}} \leqslant \mathrm{K}^{\text {hom }}\left(\beta, \mathrm{C}_{\mathrm{T}} / \mathrm{C}_{\mathrm{s}}, \lambda_{\mathrm{r}}\right)
$$

Talus homogène associé potentiellement stable.

La méthode employée pour analyser la stabilité du talus homogène associé est de type cinématique. Elle s'inspire très directement de celle utilisée par SALEN. ÇON et TRISTAN-LÓPEZ [1983] pour l'étude d'ouvrages en sols cohérents naturellement anisotropes.

On considère les mécanismes de ruine "par blocs » du talus dans lesquels la ligne de discontinuité de vitesse qui sépare le bloc en rotation du reste de l'ouvrage passe par le pied du talus (figure 7).

La fonctionnelle Phom $(v)$ calculée dans le champ de vitesse associé à un tel mécanisme s'écrit alors (voir paragraphe 2.2.2.) :

$$
\mathrm{P}^{h o m}(\underline{\mathrm{v}})=\int_{\mathrm{AB}} \pi^{\text {hom }}(\underline{\mathrm{n}} ; \underline{\mathrm{V}}) \mathrm{ds}
$$

avec :

$$
\pi^{\text {bom }}(\underline{n} ; \underline{V})=\sup \left\{-\Sigma_{1 j} n_{1} V_{1} \mid \Sigma_{1}-\Sigma_{2} \leqslant 2 C(\alpha)\right\} \text {. }
$$

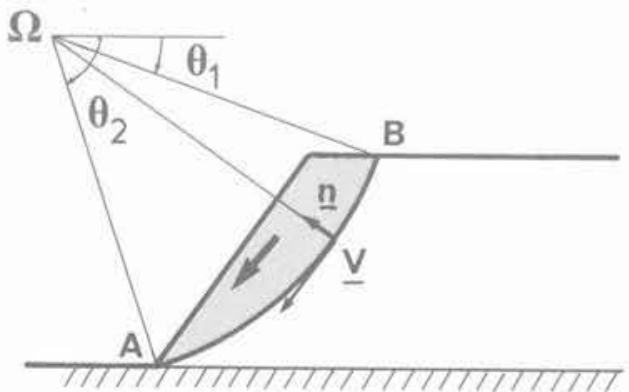

Fig. 7. - Mécanismes de ruine du talus par bloc rigide en rotation.

Fig. 7. - Rigid block failure mechanisms for the slope. 
Pour que l'approche cinématique qui découle de l'utilisation de tels champs de vitesse ne soit pas triviale, c'est-à-dire que $\mathrm{P}$ hom $(\underline{v})$ prenne une valeur finie, il est nécessaire, compte tenu du caractère purement cohérent du critère (8), que la discontinuité de vitesse $\underline{\mathrm{V}}$ soit tangentielle et donc que $A B$ soit une ligne de glissement circulaire.

Dans ces conditions l'inégalité (3) conduit, tous calculs faits, à un majorant $\mathrm{K}_{c}^{\text {hom }}$ du facteur $\mathrm{K}^{\text {hom }}$ que l'on peut écrire sous la forme :

$$
\mathrm{K}^{\operatorname{hom}}\left(\beta, r, \lambda_{r}\right) \leqslant \mathrm{K}_{\mathrm{c}}^{\text {hom }}\left(\beta, \mathrm{r}, \lambda_{\mathrm{r}}\right) .
$$
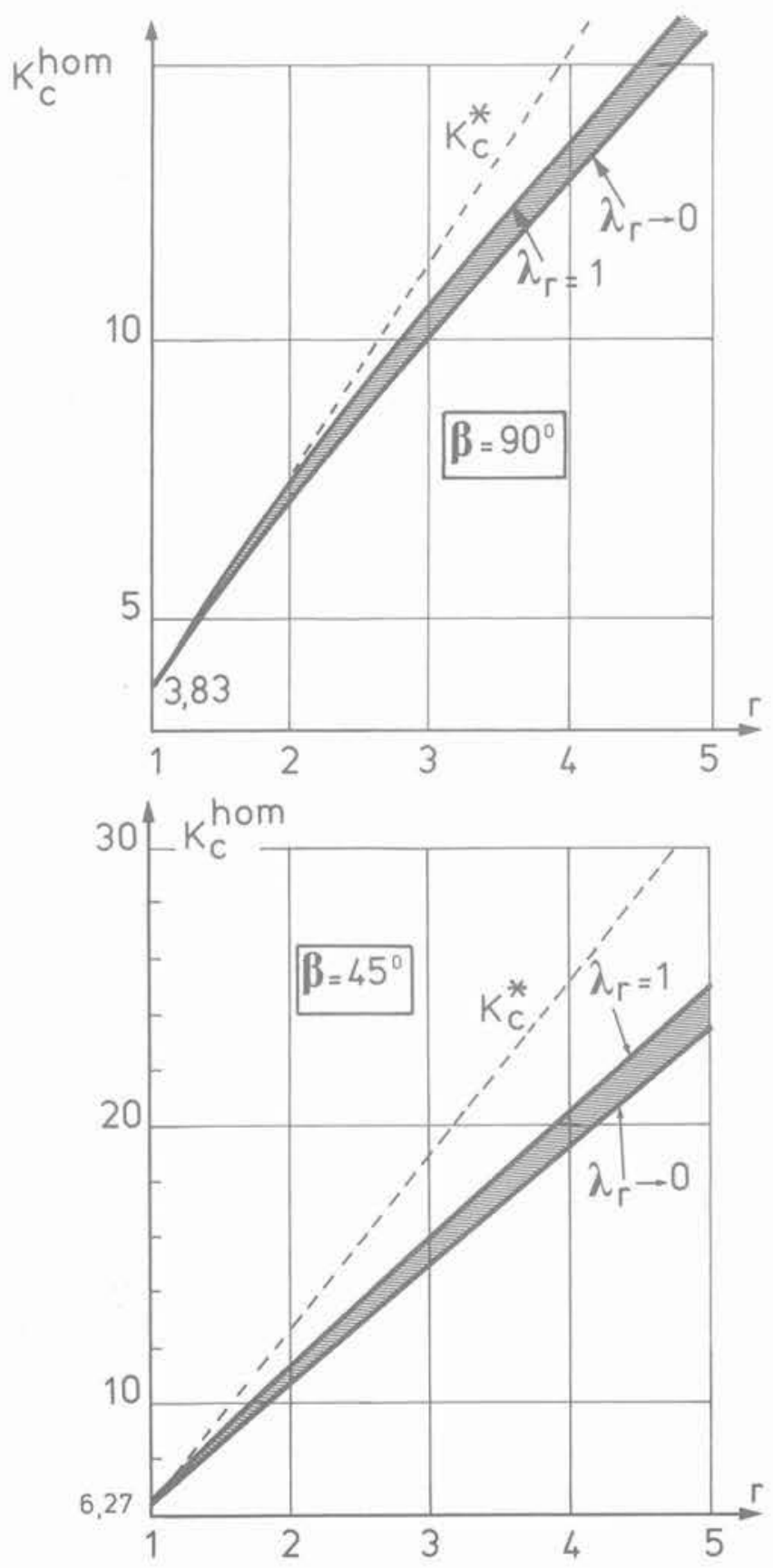

Dans cette formule

$$
\mathrm{r}=\left(\lambda_{5} \mathrm{C}_{\mathrm{s}}+\lambda_{t} \mathrm{C}_{\tau}\right) / \mathrm{C}_{\mathrm{s}}=\mathrm{C}(\alpha=0) / \mathrm{C}(\alpha=\pi / 4)
$$

est le rapport entre la cohésion maximale du sol renforcé en tant que matériau homogénéisé et celle du sol initial. Nous l'appellerons coefficient de renforcement; il est égal à 1 dans le cas du sol non renforcé. (On pourra dans (10) écrire aussi que $K^{*}$ est fonction de $\beta$, $r$, et $\lambda_{x}$ )
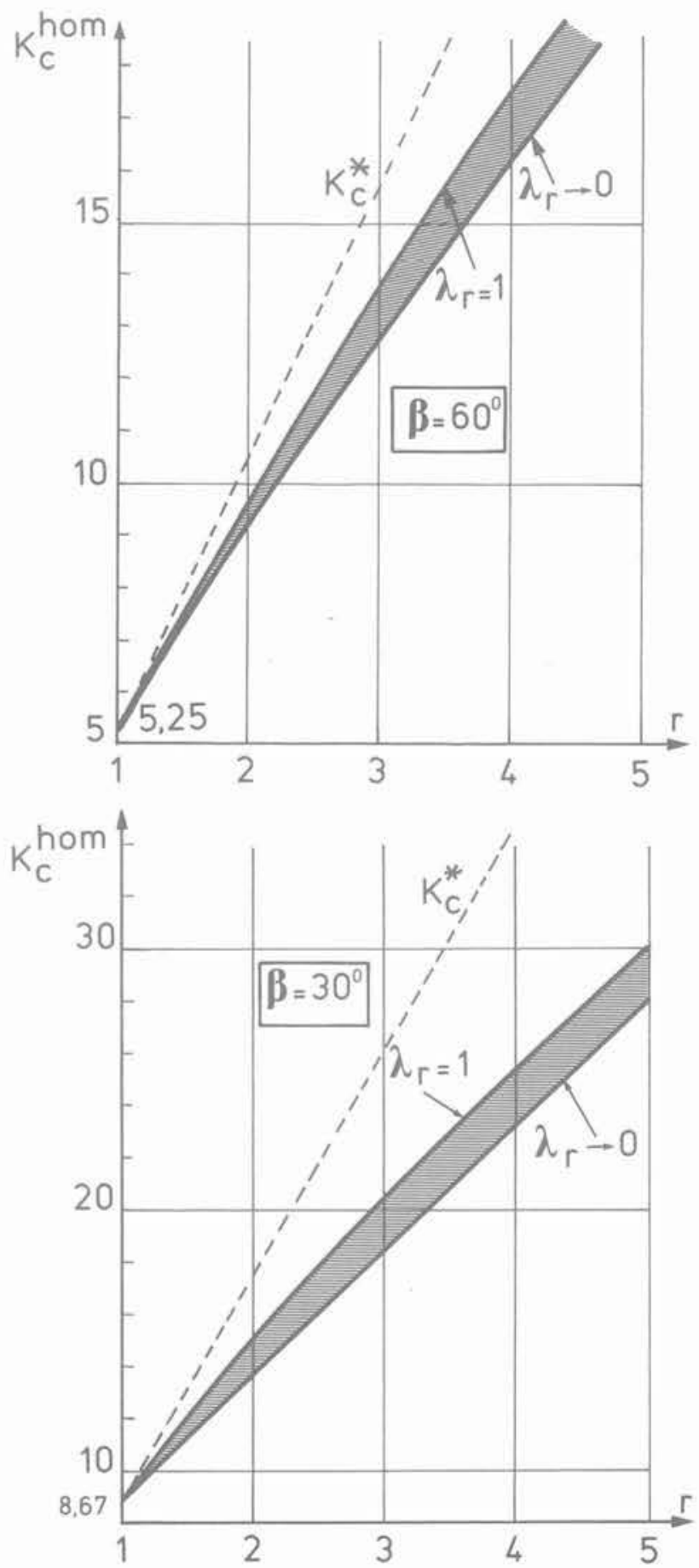

Fig. 8. - Abaques de $K_{c}^{\text {hom }}\left(\beta, r, \lambda_{r}\right)$ pour $\beta=90^{\circ}, 60^{\circ}, 45^{\circ}$ et $30^{\circ}$.

Fig. 8. - Charts for $K_{\text {hom }}(\beta, r, \lambda$, with $\beta=90^{\circ}, 60^{\circ}, 45^{\circ}$ and $30^{\circ}$. 
Les résultats obtenus par cette méthode sont représentés à la figure 8 sous forme d'abaques donnant la valeur du facteur $\mathrm{K}_{c}^{\text {hom }}$ en fonction de r et $\lambda_{T}$, pour différentes valeurs de l'angle de pente $\beta$.

\subsubsection{Comparaison avec les résultats d'une méthode cinématique directe}

L'une des approches utilisées classiquement pour le dimensionnement des pentes et talus en sols renforcés consiste à vérifier directement la stabilité de l'ouvrage initial en sol composite vis-à-vis d'une surface de rupture (représentée ici par une ligne en raison du caractère bidimensionnel du problème : figure 9) qui traverse alternativement les constituants du sol renforcé multicouche. Pour la même raison que celle invoquée précédemment dans le cas de l'ouvrage homogène associé, le caractère purement cohérent des critères de résistance des constituants du sol renforcé (et l'adhérence totale entre ceux-ci) impose à la surface de rupture d'être une surface de glissement circulaire.

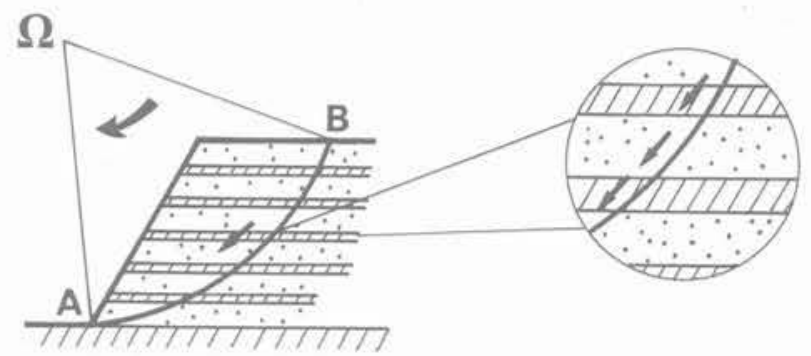

Fig. 9. - Approche cinématique directe de la stabilité du talus multicouche par cercles de glissement.

Fig. 9. - Direct kinematic approach using slip circles for the stability analysis of a multilayer embankment.

Ici encore l'approche cinématique utilisant de tels mécanismes de ruine conduit à la détermination d'un majorant de la valeur extrême $\mathrm{K}^{*}$ :

$$
K^{*}\left(\beta, r, \lambda_{r}\right) \leqslant K_{c}^{*}(\beta, r) .
$$

Ce majorant apparaît comme étant égal au produit du coefficient de renforcement $\mathrm{r}$ par une fonction $\mathrm{F}^{*}(\beta)$ représentée sur la figure 10 :

$$
\mathrm{K}_{\mathrm{c}}^{*}=r \mathrm{~F}^{*}(\beta) \text {. }
$$

On observe immédiatement que cette majoration est identique à celle qui aurait été obtenue pour un talus de pente $\beta$ constitué d'un sol homogène isotrope, dont la cohésion serait égale à $r C_{s}=\left(\lambda_{s} C_{s}+\lambda_{r} C_{r}\right)$, en utilisant la même famille de mécanismes par blocs en rotation. Les valeurs correspondantes de $\mathrm{K}_{\mathrm{c}}^{*}$ ont été reportées sur les abaques de la figure 8 (droites en pointillés) ; elles se révèlent significativement supérieures aux valeurs correspondantes du facteur $\mathrm{K}_{c}^{\text {hom : }}$

$$
\mathrm{K}_{\mathrm{c}}^{*} \geqslant \mathrm{~K}_{\mathrm{c}}^{\text {hom }} \text {. }
$$

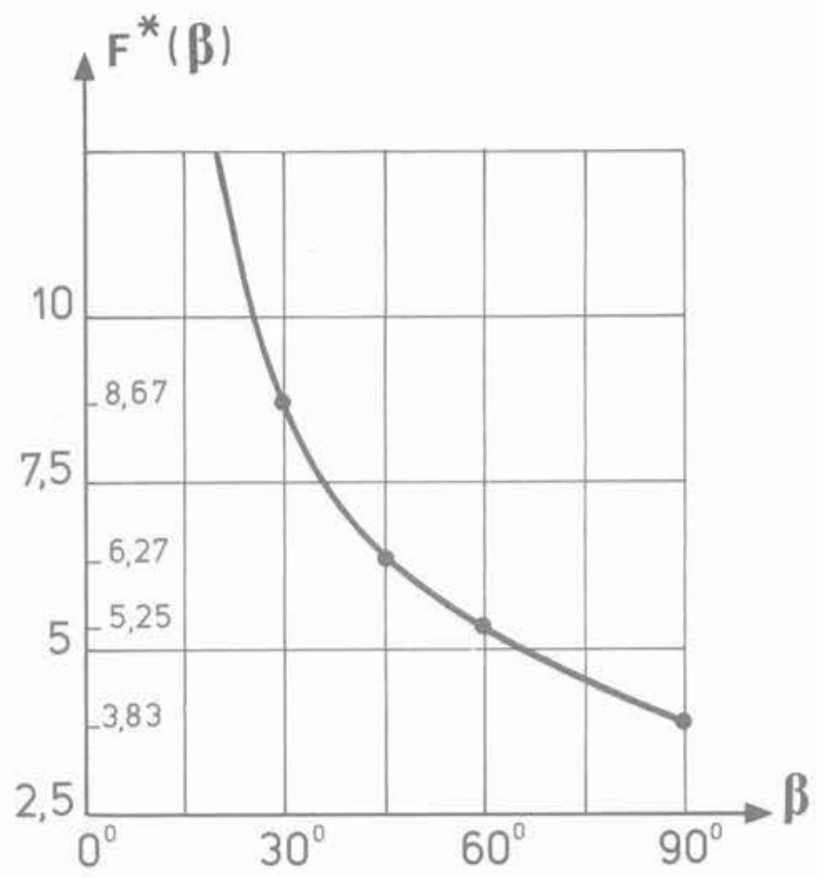

Fig. 10. - Fonction $F^{*}(\beta)$.

Fig. 10. - Function $F^{*}(\beta)$.

En combinant les inégalités (12), (13), (14) et en tenant compte du résultat d'homogénéisation qui se traduit ici par l'inégalité :

$$
\mathrm{K}^{*} \leqslant \mathrm{~K}^{\text {hom }}
$$

on aboutit alors à la succession d'inégalités :

$$
\mathrm{K}^{*} \leqslant \mathrm{~K}_{\mathrm{c}}^{\text {hom }} \leqslant \mathrm{K}_{\mathrm{c}}^{*} .
$$

Il s'agit là d'un résultat essentiel. Il signifie que pour la même famille de mécanismes de rupture (par cercles de glissement) la méthode d'homogénéisation fournit une meilleure évaluation du facteur de stabilité $\mathrm{K}^{*}$ du talus renforcé que la méthode classique appliquée directement. Cela tient fondamentalement au fait que seule la méthode d'homogénéisation, grâce à la construction explicite du critère macroscopique, est en mesure de rendre compte de l'anisotropie manifeste du sol renforcé.

\subsection{Capacité portante d'une fondation sur un massif en sol renforcé [de BUHAN - 1984]}

Les données du problème sont schématisées sur la figure 11 : semelle filante de largeur $B$ reposant sur un massif semi-infini constitué du sol renforcé multicouche défini en 3.1., et soumise à l'action d'une charge verticale centrée de densité uniforme $\mathrm{Q}$ le long de son axe de symétrie. On admettra pour simplifier que le sol est non pesant, bien que l'on puisse facilement montrer que dans le cas d'espèce, la prise en compte des forces de pesanteur ne modifie en rien la valeur de la capacité portante $\mathrm{q}^{*}$ de la fondation définie par :

$$
\mathrm{q}^{*}=\mathrm{Q}^{*} / \mathrm{B},
$$


où $Q^{*}$ désigne la valeur extrême du paramètre de chargement Q.q* peut se mettre sous la forme :

$$
\mathrm{q}^{*}=\mathrm{C}_{\mathrm{s}} \mathrm{N}^{*}
$$

où $\mathrm{N}^{*}$ est un facteur sans dimension qui ne dépend que du coefficient de renforcement $r$ et des proportions volumiques des constituants du sol renforcé :

$$
N^{*}=N^{*}\left(r, \lambda_{r}\right) \text {, }
$$
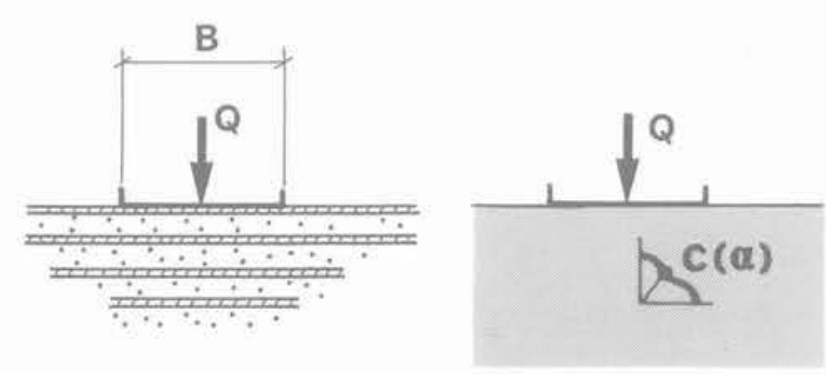

Fig. 11. - Détermination de la capacité portante d'une fondation superficielle sur sol renforcé multicouche et problème homogène associé. Fig. 11. - Determination of the bearing capacity of a footing on a multilayer reinforced soil : original and associated homogeneous problems.

De même il est facile de voir que la capacité portante de la fondation homogène associée peut s'écrire :

$$
\mathrm{q}^{\text {ham }}=\mathrm{Q}^{\text {hom }} / \mathrm{B}=\mathrm{C}_{\mathrm{s}} \mathrm{N}^{\text {hom }}\left(\mathrm{r}, \lambda_{\mathrm{r}}\right) .
$$

On recherche alors une évaluation par excès du facteur $\mathrm{N}^{\text {hom }}$ grâce à l'approche cinématique du calcul à la rupture en utilisant le schéma de ruine de Prandtl (figure 12). Ce mécanisme conduit en effet à une majoration effective de $\mathrm{N}^{\text {hom }}$ : les discontinuités de vitesse y sont tangentielles et la déformation se fait a à volume constant ( $\operatorname{tr} \mathrm{d}=0$ ) dans les secteurs circulaires $A B C$ et $A^{\prime} B^{\prime} C$, donc la fonction $P^{\text {hom }}(\underline{v})$ y prend une valeur finie.

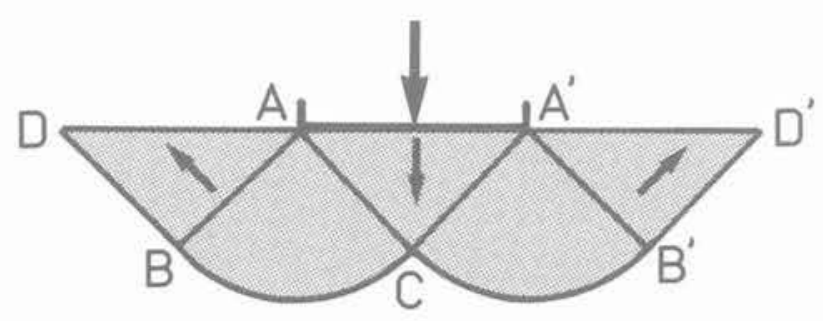

Fig. 12. - Mécanisme de Prandtl.

Fig. 12. - Prandt's mechanism.

On obtient en définitive :

$$
N^{\text {hom }}\left(r, \lambda_{r}\right) \leqslant N_{c}^{\text {hom }}\left(r, \lambda_{r}\right) \text {. }
$$

Le majorant cinématique $\mathrm{N}_{c}^{\text {hom }}$ est compris, à coefficient de renforcement $\mathrm{r}$ fixé, entre les valeurs obtenues dans les cas limites:
- $\lambda_{\mathrm{r}}=1$, couches constituées d'un matériau de cohésion $C_{\mathrm{r}}=\mathrm{r}_{\mathrm{s}}$ séparées par des interfaces de cohésion $\mathrm{C}_{\text {s; }}$;

- $\lambda_{\mathrm{r}} \rightarrow 0$, cas du renforcement par des armatures infiniment minces.

On a ainsi :

$$
N_{c}^{\text {hom }}\left(r, \lambda_{f} \rightarrow 0\right) \leqslant N_{c}^{\text {hom }}\left(r, \lambda_{r}\right) \leqslant N_{c}^{\text {hom }}\left(r, \lambda_{r}=1\right)
$$

avec les expressions explicites :

$$
\begin{aligned}
& N_{c}^{\text {horm }}\left(r, \lambda_{r} \rightarrow 0\right)=(\pi+2)+4(r-1) \\
& N_{c}^{\text {hom }}\left(r, \lambda_{r}=1\right)=(\pi+2) r+2\left[\sqrt{r^{2}-1}\right. \\
& \left.-r \operatorname{Arcos}\left(\frac{1}{r}\right)\right] .
\end{aligned}
$$

La figure 13 représente le faisceau de courbes donnant la valeur de $\mathrm{N}^{\text {hom }}$ en fonction de $\mathrm{r}$, paramétrées en $\lambda \mathrm{r}$. On y a également représenté en trait pointillé la valeur du majorant de $\mathrm{N}^{*}$ obtenu par l'approche cinématique utilisant le même mécanisme de Prandtl défini sur le massif de fondation multicouche. Ce majorant, égal à $(\pi+2) \mathrm{r}$, correspond à la valeur de la capacité portante d'une fondation sur sol homogène isotrope de cohésion égale à la moyenne pondérée des cohésions des constituants du sol renforcé.

Comme dans l'étude de stabilité du talus, on parvient à la double inégalité :

$$
N^{*}\left(r, \lambda_{r}\right) \leqslant N_{c}^{\text {hom }}\left(r, \lambda_{t}\right) \leqslant(\pi+2) r .
$$

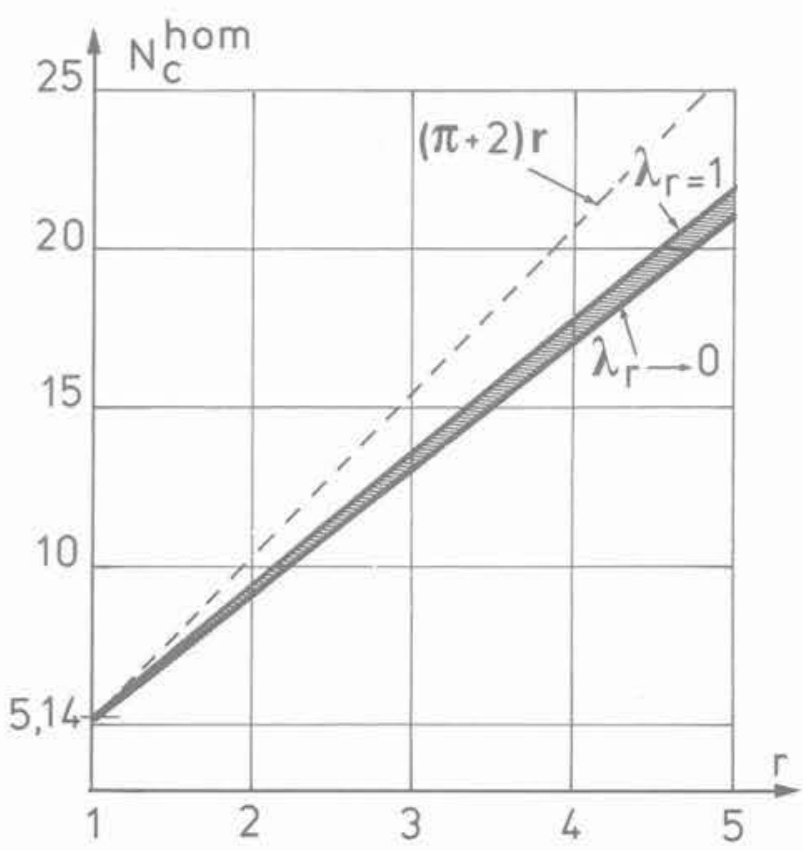

Fig. 13. - Capacité portante d'une fondation superficielle sur un sol renforcé multicouche : évaluations par la méthode d'homogénéisation et par une approche udirecte».

Fig. 13. - Bearing capacity of a surface footing on a multilayer reinforced soil : determination through the homogenization method and through a direct kinematic approach. 
On remarque là encore que la méthode d'homogénéisation conduit à une meilleure approche du facteur $\mathrm{N}^{*}$. et donc de la capacité portante de la fondation, que la méthode directe car celle-ci ne permet pas d'appréhender l'anisotropie macroscopique du sol renforcé.

\section{LA TERRE ARMÉE : UN MATÉRIAU DE TYPE «FROTTANT ANISOTROPE»}

\subsection{Critère de résistance du matériau «terre armée»}

La terre armée est ici modélisée comme un matériau multicouche bidimensionnel constitué d'un sol homogène, purement frottant (sable sec sans cohésion), obéissant à un critère de résistance de Coulomb avec un angle de frottement égal à $\varphi$, renforcé par des couches horizontales d'un matériau purement cohérent, de cohésion $C_{\text {, }}$ (figure 14)

En pratique dans le cas de la terre armée, les inclusions de renforcement sont des armatures métalliques très minces, de sorte que la proportion volumique $\lambda_{\mathrm{r}}$ est très faible (de l'ordre de $10^{-2}$ ou $10^{-3}$ ), tandis que la cohésion $\mathrm{C}_{\mathrm{r}}$ est d'un ordre de grandeur bien supérieur aux valeurs habituelles observées pour les sols. Cette configuration particulière peut s'obtenir mathématiquement en faisant tendre $\lambda$, vers zéro tout en maintenant constante la quantité $2 \lambda, C$, qui apparaît comme la résistance en traction (compression) simple des armatures rapportée à l'unité de surface transversale, et que l'on notera $\sigma^{\circ}$ :

$$
\lambda_{r} \rightarrow 0 \text { avec } \sigma^{\circ}=2 \lambda, C_{r}=\text { cte. }
$$
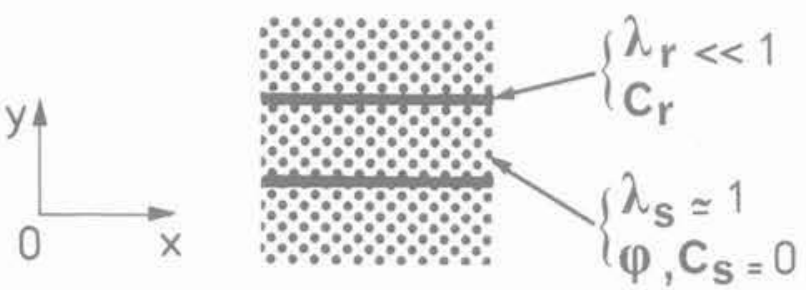

Fig. 14. - Modélisation multicouche de la terre armée.

Fig. 14. - Multilayer model for reinforced earth.

Dans ces conditions, on montre [de BUHAN - 1985], [de BUHAN et al. - 1986], que le domaine de résistance macroscopique $\mathrm{G}^{\text {hom }}$ de la terre armée est défini par:

$$
\underline{\underline{\Sigma}} \in G^{\text {hom }} \Leftrightarrow\left\{\begin{array}{l}
\underline{\underline{\Sigma}}=\underline{\sigma^{s}}+\sigma \underline{\underline{e}}_{x} \otimes \underline{\underline{e}}_{x} \\
\underline{\sigma^{s}} \in G_{s},|\sigma| \leqslant \sigma^{\circ} .
\end{array}\right.
$$

On retrouve ainsi une formulation du critère de rupture macroscopique pour un matériau renforcé par armatures très proche de celles directement postulées par Mc LAUGHLIN [1972] (matériaux renforcés par fibres) et surtout par SAWICKI [1979, 1983]. La particularité d'une telle définition réside dans le fait qu'elle ne fait plus explicitement apparaître les conditions (7) de continuité de la contrainte à l'interface sol-armature. Le matériau de renforcement n'intervient donc plus qu'à travers sa résistance en traction-compression uniaxiale dans la direction $\mathrm{Ox}$ du renforcement, rejoignant en cela l'intuition sur le « fonctionnement » pratique de la terre armée qui conduit à négliger les résistances à la flexion et au cisaillement des armatures.

La définition (25) peut d'ailleurs être complétée afin de tenir compte de la possibilité de flambement en compression des armatures. On convient alors généralement d'adopter une résistance nulle en compression, ce qui implique de modifier la définition (25) en remplaçant la condition $|\sigma| \leqslant \sigma^{\circ}$ par $-\sigma^{\circ} \leqslant \sigma \leqslant 0$.

Le domaine $G^{\text {hom }}$ ainsi obtenu est représenté sur la figure 15 dans l'espace des contraintes bidimensionnelles rapporté aux axes de coordonnées $\left(\Sigma_{x x}, \Sigma_{y y}\right.$, $\left.\sqrt{2} \Sigma_{x y}\right)$. Il s'obtient en prenant l'enveloppe convexe du cône de Coulomb $\mathrm{G}_{\mathrm{s}}$, qui caractérise la résistance du sol initial, et du cône translaté d'une quantité $-\sigma^{\circ}$ le long de l'axe des $\Sigma_{x x}$.

Cette représentation géométrique met clairement en évidence l'augmentation de résistance du sol apportée par l'introduction des armatures, puisque le domaine $\mathrm{G}_{\mathrm{s}}$ est strictement inclus dans le domaine $\mathrm{G}^{\text {hom }}$.

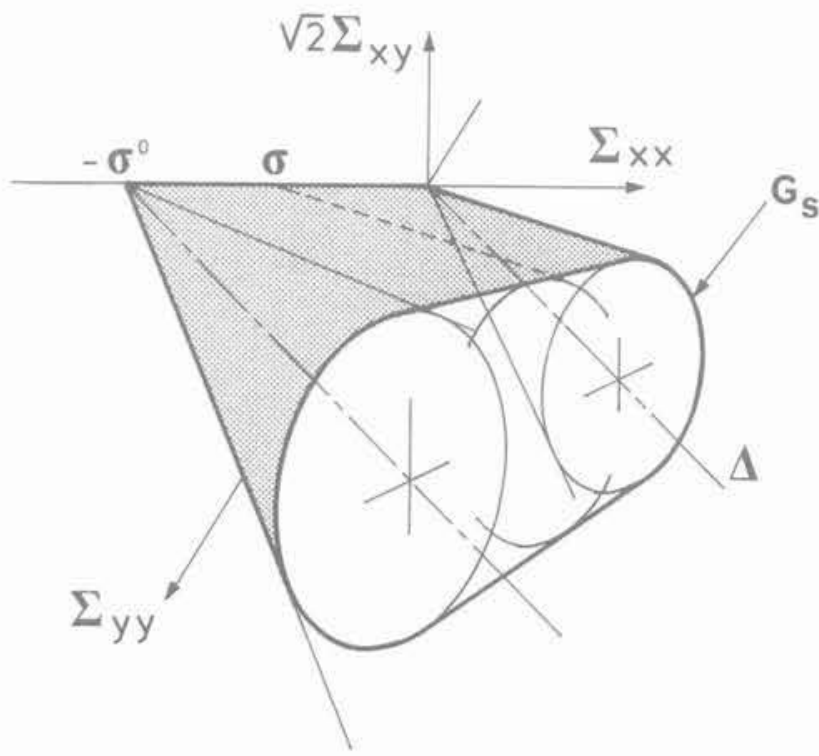

Fig. 15. - Représentation du convexe de résistance de la terre armée dans l'espace des contraintes bidimensionnelles.

Fig. 15. - The strength domain for reinforced earth drawn in the 2 - dimension stress space with coordinates $\Sigma_{x x}, \Sigma_{y y}, \sqrt{2} \Sigma_{x y}$. 
La figure 16 donne la représentation de la section du domaine $G^{\text {hom }}$ par un plan déviateur quelconque d'équation $\Sigma_{x x}+\Sigma_{y y}=$ cte, perpendiculaire à la droite $\Delta$ représentative des états de contrainte isotropes $\left(\Sigma_{x x}=\Sigma_{y y}, \Sigma_{x y}=0\right)$. Elle montre bien l'anisotropie du matériau terre armée, et plus précisément son caractère orthotrope relativement aux axes $\mathrm{Ox}$ et $\mathrm{Oy}$, car il est facile de voir qu'à l'inverse, un critère isotrope

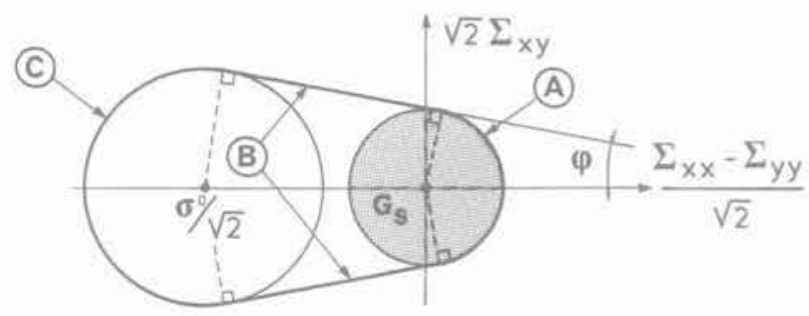

Fig. 16. - Trace du domaine $G^{\text {hom }}$ dans un plan déviateur $\Sigma_{x x}+\Sigma_{y y}=$ cte.

Fig. 16. - Section of the strength domain of reinforced earth by a deviatoric plane

$\Sigma_{x x}+\Sigma_{y y}=C t$.

aurait été représenté dans un tel plan par un cercle centré à l'origine (c'est bien le cas du critère de Coulomb) . Il convient de plus de signaler qu'un tel critère ne rentre pas dans le cadre de ceux étudiés par BOEHLER et SAWCZUK [1970] en se fondant sur la notion de «tenseur d'anisotropie ».

Cette représentation géométrique permet en outre de distinguer trois « modes de rupture » pour le matériau terre armée qui correspondent à des façons différentes d'atteindre la frontière du domaine de résistance $G^{\text {hom }}$, que l'on peut interpréter comme suit :

- pour les points de type A communs aux frontières de $G^{\text {tom }}$ et de $G_{5}$, la résistance du sol est mobilisée, tandis que les armatures sont à la limite de leur résistance en compression $(\sigma=0)$;

- les points de type B correspondent aux états de contrainte macroscopique pour lesquels la résistance du sol est mobilisée, tandis que les armatures supportent un effort de traction inférieur à leur limite de résistance $\left(-\sigma^{\circ}<\sigma<0\right)$;

- enfin pour les points de type $\mathrm{C}$ communs aux frontières de $\mathrm{G}^{\text {hom }}$ et du cône translaté, la résistance du sol est là encore mobilisée, tandis que les armatures ont atteint leur limite de résistance en traction $\left(\sigma=-\sigma^{\circ}\right)$.

\subsection{Confrontation avec des résultats expérimentaux}

La comparaison du critère ainsi construit par voie théorique avec des résultats d'essais à l'appareil « triaxial » effectués sur des échantillons de sable armé par des disques métalliques selon différentes inclinaisons [LONG et URSAT - 1977] (figure 17), a été faite par MANGIAVACCHI et PELLEGRINI [1985].

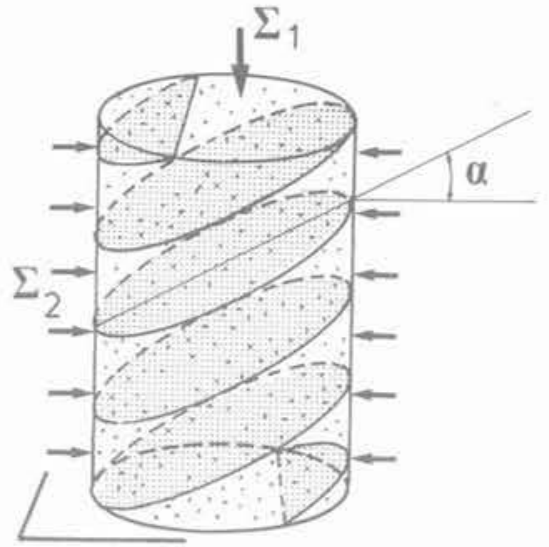

Fig. 17. - Schématisation d'un essai "triaxial» sur des éprouvettes de sable armées par des disques métalliques.

Fig. 17. - Triaxial test performed on sand specimens reinforced by metallic sheets.

Il est commode, afin de rendre cette comparaison plus facile, d'exprimer le critère de résistance macroscopique (25) (modifié pour tenir compte de la résistance nulle des armatures en compression) en fonction des contraintes principales $\Sigma_{1} \geqslant \Sigma_{2}=\Sigma_{3}$ et de l'inclinaison $\alpha$ des renforcements :

$$
\begin{aligned}
& \underline{\Sigma} \in \mathrm{G}^{\text {hom }} \Leftrightarrow \\
& {\left[\left(\Sigma_{1}-\Sigma_{2}\right)^{2}+2 \sigma\left(\Sigma_{1}-\Sigma_{2}\right) \cos 2 \alpha+\sigma^{2}\right]^{1 / 2}} \\
& \leqslant\left(\Sigma_{1}+\Sigma_{2}-\sigma\right) \sin \varphi \\
& \text { avec }-\sigma^{\circ} \leqslant \sigma \leqslant 0 \text {. }
\end{aligned}
$$

Il est alors possible, pour chaque valeur de l'angle $\alpha$, de tracer la courbe de rupture correspondante dans le plan $\left(\Sigma_{1}, \Sigma_{2}\right)$. Cette courbe est obtenue comme l'enveloppe d'une famille d'hyperboles paramétrée en

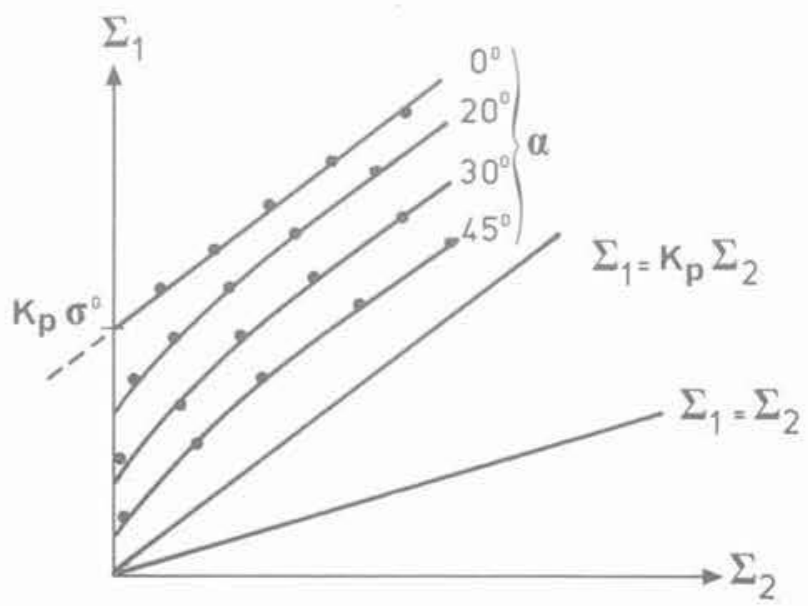

Fig. 18. - Courbes de rupture pour le sable armé, $\left(\varphi=38^{\circ}, \varphi^{\circ}=28,7 \mathrm{kPa}\right)$

dans le plan $\left(\Sigma_{1}, \Sigma_{2}\right)\left(K_{p}=\operatorname{tg}^{2}(\pi / 4+\varphi / 2)\right)$.

Fig. 18. - Failure curves for a reinforced sand drawn in the $\left(\Sigma_{1}, \Sigma_{2}\right)$ plane $\left(K_{\rho}=\tan ^{2}(\pi / 4+\varphi / 2)\right)$. 
$\sigma \in\left[-\sigma^{\circ}, 0\right]$. Elle peut également s'interpréter l'espace des contraintes par le plan passant par la droite $\Delta$ et inclinée d'un angle $2 \alpha$ par rapport au plan $\Sigma_{\mathrm{xy}}=0$.

La figure 18 montre quelques-unes de ces courbes théoriques calculées à partir des caractéristiques de résistance du sol $\left(\varphi=38^{\circ}\right)$ et des renforcements $\left(\sigma^{\circ}=28.7 \mathrm{kPa}\right)$ utilisés dans les essais triaxiaux. Il ressort du simple examen de cette figure, sur laquelle les points expérimentaux ont également été reportés, que l'accord entre la modélisation théorique proposée et les données expérimentales est excellent, dans tout le $\left(\Sigma_{1} \geqslant \Sigma_{2} \geqslant 0\right)$. comme la section de la frontière du domaine $G$ hom dans domaine de contrainte exploré dans ces expériences

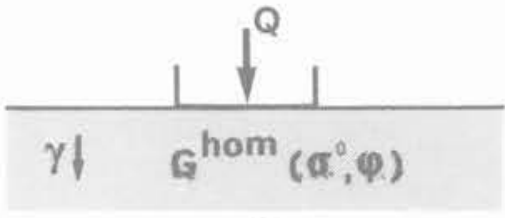

(a)

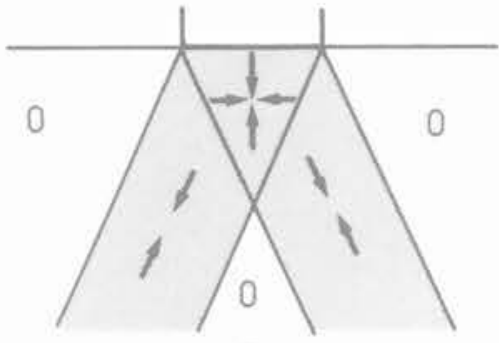

(b)

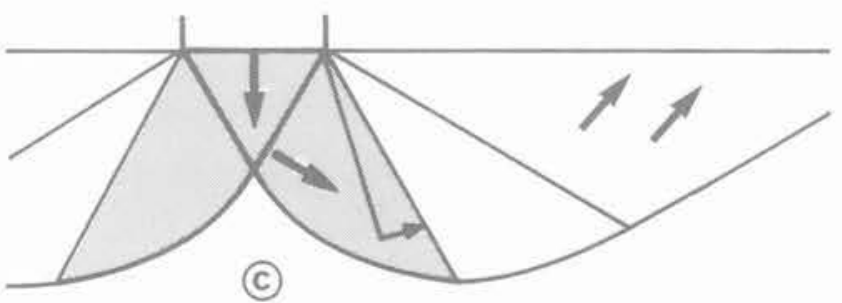

Fig. 19. - (a) Problème homogène associé. (b) Champ statique.

(c) Champ cinématique de Prandtl.

Fig. 19. - (a) Associated homogeneous problem. (b) Stress field.

(c) Prandtl kinematic field.

effets de la pesanteur et du renforcement du sol en allant dans le sens de la sécurité

$$
q^{\text {ham }} \geqslant 1 / 2 \gamma B N_{\gamma}(\varphi)+\sigma^{0} N_{\uparrow}(\varphi) .
$$

Dans cette formule, $\mathrm{q}^{\text {hom }}=\mathrm{Q}^{\text {hom }} /$ B est la capacité portante de la fondation, $\gamma$ le poids volumique du matériau terre armée, tandis que $N_{-}(\varphi)$ et $N_{,}(\varphi)$ représentent respectivement le coefficient classique du terme de surface calculé pour un sol homogène pesant régi par le critère de Coulomb, et un coefficient sans dimension correspondant au sol renforcé supposé non pesant.

L'évaluation de $\mathrm{N}_{70}$ se fait en mettant en ceuvre les approches statique et cinématique du calcul à la rupture.

Ainsí, en optimisant le champ statique représenté sur la figure $19 \mathrm{~b}$, on trouve :

$$
\begin{aligned}
& N_{\circ \circ}(\varphi) \geqslant \cos ^{2} \varphi /(1-2 \sin \varphi) \text { si } \sin \varphi \leqslant 1 / 4 \\
& N_{\gamma \circ}(\varphi) \geqslant\left(1+\sqrt{\sin \varphi)^{2}}(1+\sin \varphi) / 2(1-\sin \varphi)\right. \\
& \text { si } \sin \varphi \geqslant 1 / 4
\end{aligned}
$$

(La deuxième expression de (28) a été donnée par [SAWICKI - 1983].)
L'utilisation de l'approche cinématique nécessite le calcul préalable des fonctions $\alpha \pi$ » relatives au domaine de résistance $G^{\text {hom }}$ défini par (25) (avec $\left.-\sigma^{\circ} \leqslant \tau \leqslant 0\right)$. Soit :

$\pi^{\text {hom }}(\mathrm{d})=\left\{\begin{array}{l}r^{\circ} \sup ^{\prime} \mathrm{d}_{\mathrm{xx}} \cdot 0 ; \text { si } \operatorname{tr}(\mathrm{d}) \geqslant\left(\left|\mathrm{d}_{1}\right|+\left|\mathrm{d}_{2}\right|\right) \sin \varphi \\ +\infty \text { sinon }\end{array}\right.$ $\left(d_{1}\right.$ et $d_{2}$ sont les composantes principales de $d$ ); ainsi que :

$\pi^{\text {horm }}(\underline{n}: \underline{V})=\left\{\begin{array}{l}\sigma^{\circ} \sup \mid V_{x} n_{x} \cdot 0 ; \text { si } \underline{V} \cdot \underline{n} \geqslant|\underline{V}| \sin \varphi \\ +\infty \text { sinon. }\end{array}\right.$

On remarque à partir des expressions précédentes, que les conditions portant sur $\underline{\underline{d}}$ ou $(\underline{n} ; \underline{V})$ pour que les fonctions $\pi^{\text {hom }}$ prennent des valeurs finies sont les mêrmes que celles valables pour le sol non renforcé obéissant au critère de Coulomb. Il est par conséquent possible d'utiliser, dans le cadre de l'approche cinématique, le champ de vitesse de Prandtl (figure 19c) qui permet alors d'obtenir la majoration suivante

$$
\mathrm{N}_{\pi} \circ(\varphi) \leqslant(1+\sin \varphi) \exp [(\pi / 2+\varphi) \operatorname{tg} \varphi] .
$$


Dans ce champ de vitesse, les fonctions $\pi^{\text {hom }}$ explicitées ci-dessus ne prennent des valeurs non nulles que dans les zones grisées, ainsi que le long des lignes de discontinuité de vitesse renforcées sur la figure.
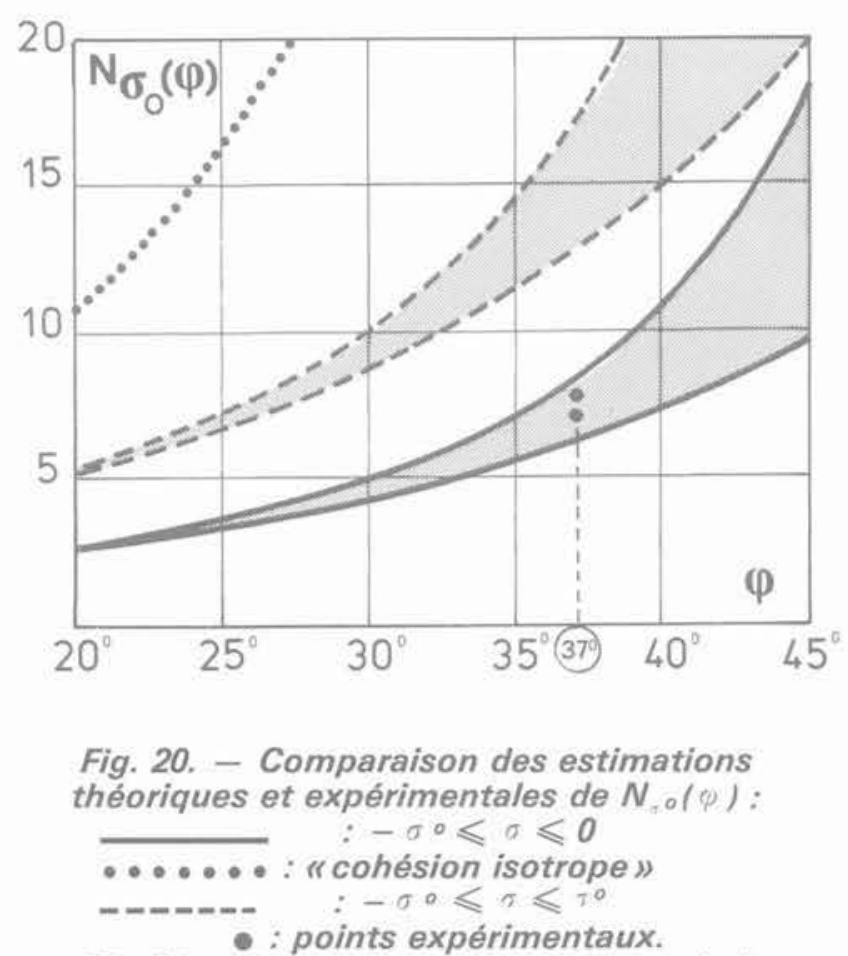

Fig. 20. - Comparison between theoretical and experimental estimates of $N_{o}(\varphi)$ :

$\begin{aligned} \cdots . \cdots \cdot & : \text { "isotropic cohesion } \| \\ i C_{\text {iso }} & \left.=\sigma^{\circ} \cdot \tan (\pi / 4+\varphi / 2) / 2\right) \\ & : \text { experimental results. }\end{aligned}$

Les courbes correspondant à l'encadrement de $\mathrm{N}_{n 0}(\varphi)$ ainsi obtenu sont représentées sur la figure 20 . On y a également représenté les résultats expérimentaux rapportés par SAWICKI [1983] concernant la réalisation d'expériences sur modèles réduits de poinçonnement d'un massif en terre armée (sable avec un angle de frottement de $\varphi=37^{\circ}$. et pour différentes valeurs de $\sigma^{\circ}$ ) que l'on a interprétés à travers la formule de superposition (27). On constate que ces résultats s'inscrivent parfaitement dans le faisceau délimité par les approches statique et cinématique, dans l'hypothèse de résistance nulle en compression des armatures.

A titre de comparaison, nous avons également reporté sur la figure 20 :

- le faisceau obtenu en supposant que les armatures peuvent également travailler en compression jusqu'à la limite $\sigma^{\circ}$ :

- la courbe en pointillé qui correspond à un calcul fait dans l'hypothèse où l'on modélise la terre armée comme un matériau de Coulomb d'angle de frottement $\varphi$ et de cohésion isotrope $\mathrm{C}_{\mathrm{iso}}=\left(\pi^{\circ} / 2\right) \operatorname{tg}(\pi / 4+\varphi / 2)$. Le domaine de résistance correspondant dans l'espace des contraintes est le plus petit cône de Coulomb qui contienne le domaine $\mathrm{G}^{\text {hom. }}$. Ce dernier type de calcul conduit, ainsi que STÉFANI et LONG [1979] l'avaient déjà indiqué, à une évaluation beaucoup trop optimiste de la capacité portante. On peut montrer en revanche [SIAD - 1987], qu'en ce qui concerne l'analyse de stabilité d'un mur de soutènement en terre armée, une telle * approximation d'isotropie » donne des résultats à peine supérieurs à ceux de la méthode d'homogénéisation qui prend en compte lanisotropie de la terre armée : elle peut donc dans ce cas constituer une méthode de dimensionnement acceptable.

La position des points expérimentaux par rapport à ces deux dernières évaluations théoriques vient conforter la validité de l'approche par la méthode d'homogénéisation en calcul à la rupture, et tend par ailleurs à prouver que l'hypothèse faite au départ de résistance nulle des armatures en compression, rend bien compte du fonctionnement de ces dernières dans la terre armée.

\section{CONCLUSION}

Comme on s'est efforcé de le montrer à travers les quelques exemples traités dans cet article, la méthode d'homogénéisation élaborée dans le cadre de la théorie du calcul à la rupture constitue un outil adéquat pour le dimensionnement des ouvrages en sols renforcés. Simple dans sa conception, sa mise en ceuvre repose sur la possibilité de déterminer le critère de résistance du sol renforcé considéré en tant que matériau homogène, à partir de ceux de ses constituants. C'est ainsi que l'on a pu obtenir sur la base d'une modélisation multicouche. une construction explicite du critère de résistance de la terre armée, lequel apparaît comme étant du type « frottant anisotrope». Cette même modélisation permet en outre. par une simple modification dans l'écriture du critère macroscopique, de prendre en compte n'importe quelle condition de frottement à l'interface entre le sol et les armatures (voir [de BUHAN - 1985. 1986])

Une telle méthode est susceptible de s'appliquer à bien d'autres types d'ouvrages en sols renforcés, qu'il s'agisse des procédés de renforcement des sols par clouage, par l'introduction de micropieux, ou des techniques de traitement en place par colonnes. Mais il va de soi que son efficacité au plan pratique dépend très directement de la détermination numérique du critère macroscopique. Or, en raison du caractère tridimensionnel de la plupart de ces modes de renforcement, ce critère ne peut plus s'obtenir à partir de formulations analytiques aussi simples que dans le cas du modèle multicouche, mais nécessite, ainsi que nous l'avons vu, la résolution d'un problème de calcul à la rupture défini sur la cellule de base représentative de la structure du sol renforcé. Il importe néanmoins de ne pas perdre de vue que la résolution d'un tel problème ne constitue, dans la mise en cuure de la méthode d'homogénéisation, qu'une étape de calcul intermédiaire. Il conviendra donc dans la mesure du possible, de rechercher des formulations analytiques approchées du critère macroscopique, mieux adaptées à la résolution dans un deuxième temps du problème homogène associé.

Quoi qu'il en soit, on peut raisonnablement s'attendre. 
sur la base des premiers résultats obtenus, à ce que la méthode d'homogénéisation donne lieu dans les prochaines années à des développements importants dans le domaine du dimensionnement des ouvrages en sols renforcés.

\section{BIBLIOGRAPHIE}

Colloque international sur le renforcement des sols : terre armée et autres techniques (1979), Paris, Ass. Anc. El. de l'E.N.P.C., vol. I à III.

Renforcement en place des sols et des roches (1984). Comptes rendus du Colloque International, Paris, 9-11 octobre. Presses de l'E.N.P.C.

Comptes rendus du XIe Congrès International de Mécanique des Sols et Travaux en Fondations (1985). San Francisco.

BASTICK M. (1987), L'apport de la méthode des éléments finis à l'étude du comportement des ouvrages en terre armée. Journées d'études sur l'utilisation de la méthode des éléments finis en géotechnique, Paris.

BLONDEAU F., CHRISTIANSEN M., GUILLOUX A., SCHLOSSER F. (1984), Talren : méthode de calcul des ouvrages en sols renforcés. Coll. Int. Renf. des Sols en place, Paris, Presses de l'E.N.P.C., pp. 219 224.

BOEHLER J.P., SAWCZUK A. (1970), Équilibre limite des sols anisotropes. Jl de Mécanique, vol. IX, $\mathrm{n}^{\circ} 1$, pp. 5-33.

BROWN B.S., POULOS H.G. (1979). Analysis of Foundations on Reinforced Soil. C.R. Xe Cong. Int. Méc. Sols. Stockholm, pp. 595-598.

De BUHAN P. (1984), Détermination de la capacité portante d'une fondation sur sol renforcé par une méthode d'homogénéisation. C.R. Coll. Int. Renf. Sols en place, Presses de l'E.N.P.C.. Paris, pp. 127. 132.

De BUHAN P. (1985), Calcul par une méthode d'homogénéisation de la résistance au poinçonnement d'un demi-espace multicouche, VIle Cong. Fr. de Méc., Bordeaux.

De BUHAN P. (1985), Critère de rupture macroscopique d'un matériau renforcé par armatures. C.R. Ac. Sc. Paris, t. 301, série 11, pp. 557-560.

De BUHAN P. (1986), Approche fondamentale du calcul à la rupture des ouvrages en sols renforcés. Thèse d'État, Université Pierre et Marie Curie, Paris.

De BUHAN P., SALENÇON J. (1985), Analyse de stabilité d'ouvrages en sols renforcés. C.R. Xle Cong. Int. Méc. Sols, San Francisco, pp. 1749-1752.

De BUHAN P., SALENÇON J., SIAD L. (1986), Critère de résistance pour le matériau "terre armée». C.R.Ac.Sc., Paris, t. 302, série Il, pp. 377-381.

CISS A. (1985), Étude de la stabilité des massifs en sol renforcé par des armatures métalliques à l'aide de formulations numériques des méthodes de lanalyse limite. Thèse de Docteur-Ingénieur, Université de Grenoble.

CORTE J.F. (1977), La méthode des éléments finis appliquée aux ouvrages en terre armée. Bull. Liaison Labo. P. et Ch., 90, pp. 37-48.

DELMAS Ph., BERCHE J.C., CARTIER G., ABDELHEDI A. (1986), Une nouvelle méthode de dimensionnement du clouage des pentes: programme PROSPER. Bull. Liaison Labo. P. et Ch., 141, PP. 57-66

GERRARD Ch. (1982), Reinforced soil : an orthorombic material. Jl of Geotechnical Eng. Div., A.S.C.E. vol. 108,11 , pp. 1460-1474.

GERRARD Ch., PANDE G.N., SCHWEIGER H.F. (1984). Modelling behaviour of soft clays reinforced with stone columns. Coll. Int. Renf. des Sols en place, Paris, Presses de l'E.N.P.C., pp. 145-150.

JURAN 1., SCHLOSSER F. (1979), Étude theorique des efforts de traction dans les armatures des ouvrages en terre armée. Coll. Int. Renf. des Sols, Paris, pp. 77-82.

JURAN I., SHAFFIEE S., SCHLOSSER F. (1985), Les soutènements par clouage. Étude sur modèles numériques. C.R. Xle Cong. Int. Méc. Sols, San Francisco, pp. 1713-1716.

LEFLAIVE E. (1985), Sol renforcé par des fils continus : le Texsol. C.R. XIe Cong. Int. Méc. Sols, San Francisco, pp. 1787-1790.

LESHCHINSKY D., REINSCHMIDT A.J. (1985), Stability of membrane reinforced slopes. Jl of Geotech. Eng., A.S.C.E., vol. 111, n ${ }^{\circ} 11$, pp. $1285-1300$.

LONG N.T., URSAT P. (1977), Comportement du sol renforcé. Rapport de recherche, L.P.C. Strasbourg.

LONG N.T., GUEGAN Y., LEGEAY G. (1972), Étude de la terre armée à l'appareil triaxial. Rapport dé recherche des L.P.C.

Mc LAUGHLIN P.V. (1972), Plastic limit behavior and failure of filament reinforced materials. Int. Jl Solids and Structures, vol. $8, \mathrm{n}^{\circ} 11, \mathrm{pp} .1299-1318$.

MANGIAVACCHI R., PELLEGRINI G. (1985), Analisi teorica del comportamento della terra armata mediante una procedura di omogeneizzazione. Tesi di Laurea, Politecnico di Milano.

PASTOR J., TURGEMAN S., CISS A. (1986), Calculation of limit loads of structures in soils with metal reinforcement. Proc. European Conference on Numerical Methods in Geomechanics, Stuttgart.

RENOUF A., IORIO J.P., BESANÇON G. (1982), Stabilisation d'une pente marine bicouche par vibration profonde. Revue Française de Géotechnique, $n^{\circ} 18$, pp. 67-77.

SALENÇON J. (1973), Théorie de la plasticité pour les applications à la mécanique des sols. Eyrolles, Paris.

SALENÇON J. (1983), Calcul à la rupture et analyse limite. Presses de l'E.N.P.C., Paris. 
SALENÇON J., TRISTAN-LOPEZ A. (1983), Calcul à la rupture en mécanique des sols : cas des sols cohérents anisotropes. Annales de II.T.B.T.P., 413, pp. 53-83.

SAWICKI A. (1979), Continuum theory of reinforced earth. Proc. Coll, Franco-Polonais, Paris, pp. 53-70.

SAWICKI A. (1983), Plastic Limit Behavior of Reinforced Earth. Jl of Geotechnical Eng. Div., A.S.C.E., vol. $109, n^{\circ} 7$, pp. 1000-1005.
SIAD L. (1987), Dimensionnement d'ouvrages en terre armée par une méthode d'homogénéisation. Thèse de doctorat E.N.P.C., Paris. A paraître.

STEFANI C., LONG N.T. (1979), Comportement de semelles sur un massif armé semi-infini. C.R. Coll. Int. Renforcement des Sols, Paris, pp. 185-190.

SUQUET P. (1983), Analyse limite et homogénéisation. C,R. Ac, Sc. Paris, t. 296, série II, pp. 13551358. 